Espacio, Tiempo y Forma, Serie VII, H. ${ }^{a}$ del Arte, t. 13, 2000, págs. 271-¿92

\title{
I funerali di Carlo III nella cattedrale di Palermo
}

\author{
GiUsePPINA LEONE
}

SINTESI ABSTRACT

Nel 1789 in occasione del funerale di Carlo III re delle Spagne e dell'infante di Napoli,

D. Gennaro Borbone viene pubblicato a Palermo a cura della Reale Stamperia un interessante volume che illustra le cerimonie svolte in tale occasione nonché il progetto effimero elaborato per la navata principale della cattedrale di Palermo. L'architetto incaricato per il progetto è il sacerdote ingegnere

Salvatore Attinelli. Motivo di sorprendente interesse è il fatto che l'intervento si colloca in un cantiere aperto. Infatti, ventidue anni prima, nel 1767, era stato commissionato dall'arcivescovo Serafino Filangeri un progetto a Ferdinando Fuga per il totale rinnovamento della prestigiosa fabbrica palermitana. II progetto effimero proposto da Attinelli (che lavora da anni nel cantiere della cattedrale) sembra costituire pretesto per un ultimo tentativo di revisione del progetto in corso di cantiere. Sembra cioè collocarsi sulla scia delle polemiche nei confronti del
In 1789 on occasion of the funerals of Carlo III, king of Spain, and of the Infante of Naples, $D$. Gennaro Borbone, it was published in Palermo by the Royal Printing-house an interesting volume illustrating the ceremonies which have been carried out in this event, as well as the ephemeral project elaborated for the nave of Palermo cathedral. The architect uncharged of the project was Salvatore Attinelli a priest engineer. It's ground of surprising interest the fact that this happened while the building was in construction. As matter of fact, twenty-two years before, in 1767, a project had been ordered to Ferdinando Fuga by the archbishop Serafino Filangeri for a complete renewal of the prestigious building of Palermo. The ephemeral project proposed by Salvatore Attinelli (who had been working at the construction of the cathedral for many years) seems to be a pretext for a last attempt to review a project in progress. So it seems to constitute a polemic towards 
progetto del Fuga, che aveva ricevuto tante critiche da dover essere sottoposto all'approvazione

reale. Lo studio di questo apparato effimero offre dunque uno spunto di riflessione sulle cerimonie funebri che venivano colte come occasione per modificare le scelte architettoniche intraprese.
Fuga's project; this plan had received so many critical opinions to be exposed for a royal approval. The study of this ephemeral project offers a starting point of reflection about real meaning of these funeral ceremonies which become occasions to modify the architectonic choices already undertaken

II 13 agosto 1789 viene pubblicata, a cura della Reale Stamperia, su commissione dei Consiglieri del Real Patrimonio una relazione delle pompe funebri celebrate a Palermo in onore di Carlo III, re delle Spagne, e di D. Gennaro Borbone, Infante di Napoli.

La descrizione delle esequie è affidata a Giovanni Evangelista Di Blasi, «regio istoriografo»; le orazioni funebri sono curate dal P. Camillo Di Maria delle scuole pie e da P. D. Saverio Granata; le iscrizioni latine sono dell'erudito P. Francesco Murena. La splendida edizione, adorna di anteporta firmata dal pittore Giuseppe Velasquez, contiene cinque eleganti tavole di grandi dimensioni incise da Giovanni Gramignani.

L'eccezionalità di questo ormai raro libro non si deve considerare soltanto in relazione all'accuratezza e ricercatezza della pubblicazione, che rientra nella produzione letteraria del tradizionale filone della narrativa funebre, quanto nel suo offrire un interessante e nuovo spunto di lettura al lento ed interminabile cantiere della Cattedrale di Palermo che nel 1789, in occasione del funerale di Carlo III, contro ogni ragionevole aspettativa ${ }^{2}$, viene prescelta

II 14 dicembre del 1788 muore a Madrid Carlo III. Quando Ferdinando riceve la notizia della morte del padre e della successione al trono del fratello Carlo IV, il suo regno è già a lutto per la morte del piccolo Infante di Napoli, D. Gennaro Carlo. La prima preoccupazione del re delle Due Sicilie è di ordinare che funerali solenri siano celebrati per tutto il regno. «Prendere il lutto per sei mesi, cioè ne' primi due rigorosissimo con rattina, e il primo di essi senza polvere, e senza manichetti, ne' due secondi con panno con poche asole, come si costuma nel lutto rigoroso, e negli ultimi due più leggiero. Ha inoltre la M.S. risoluto, che si chiudano li Teatri, e restino sospesi gli spettacoli fino a nuovo ordine». G.E. DI BLASi E Gambacorta, Relazione delle Pompe Funebri ordinate in Palermo Da' Ministri del Real Patrimonio, in Funerali per Carlo lll Re delle Spagne e per I'Infante di Napoli D. Gennaro Borbone. Palermo 1789, pag. 23.

2 «Purtuttavia contro la comune aspettativa prescelto videsi l'antico Duomo...si stranizzò ognuno del popolo nel vedere pensare una cosa che veramente era sorta senza discorso e senza riuscita. E pure cossi si ebbe a fare...». F. M. EMANUELE E GAETANI, marchese di Villabianca, Le feste reali di Sicilia nel secolo XVIII, a cura di M. C. RugGIERI ThICOLI, Palermo 1991, pag. 191. Si noti invece come lo storiografo ufficiale riferisca diversamente sulle reazioni che la cittadinanza di Palermo manifesta per la decisione di celebrare i funerali di Carlo 111 in Cattedrale: "Grande fu allora la sorpresa per 


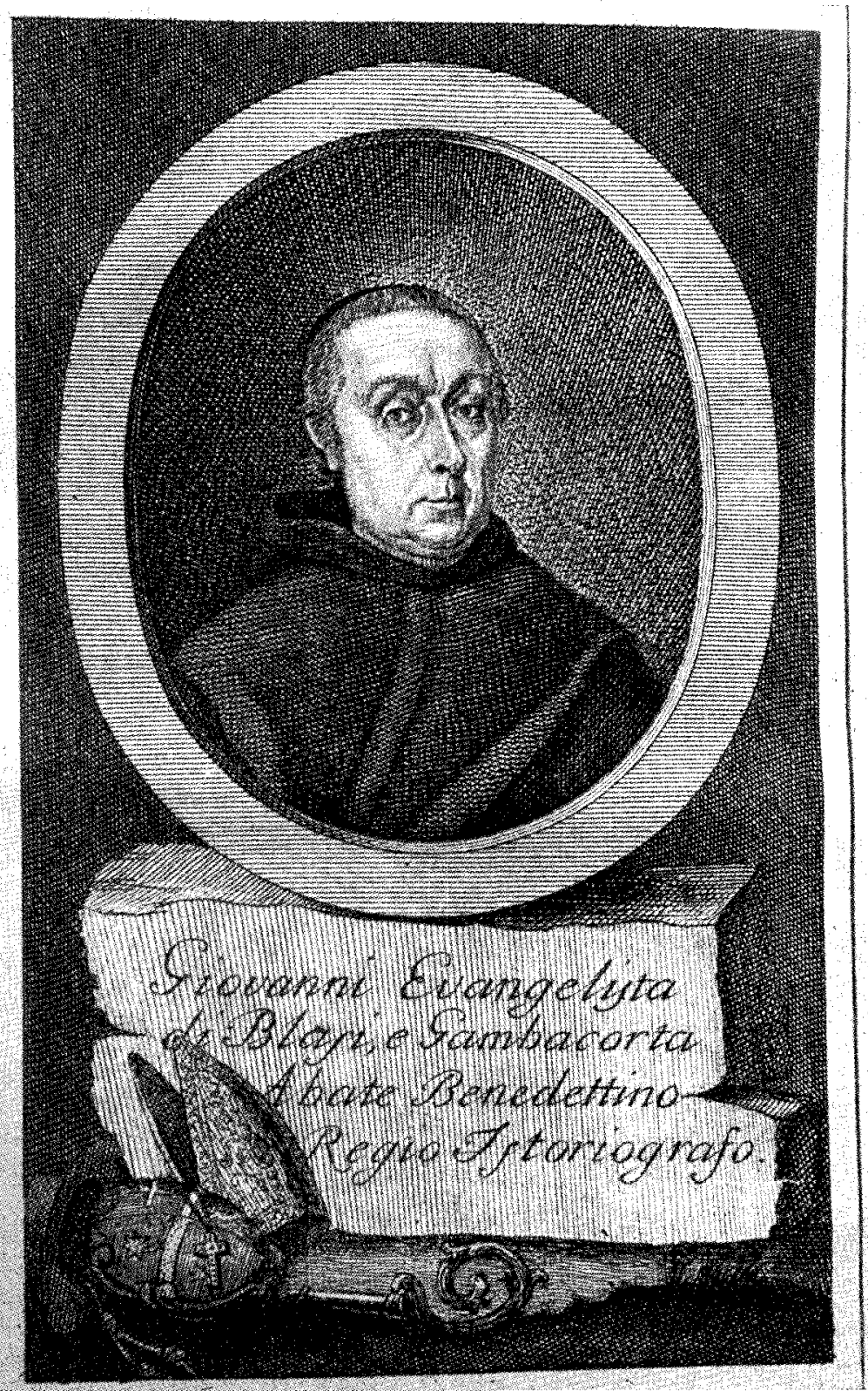

Fig. 1. Giovanni Evangelista Di Blasi e Gambacorta «Regio Istoriografo», ritratto, incisione di Giovanni Gramignani, (in: G. E. Di Blasi e Gambacorta Storia cronologica de' Viceré, luogotenenti, e presidenti del Regno di Sicilia, Palermo 1791). 


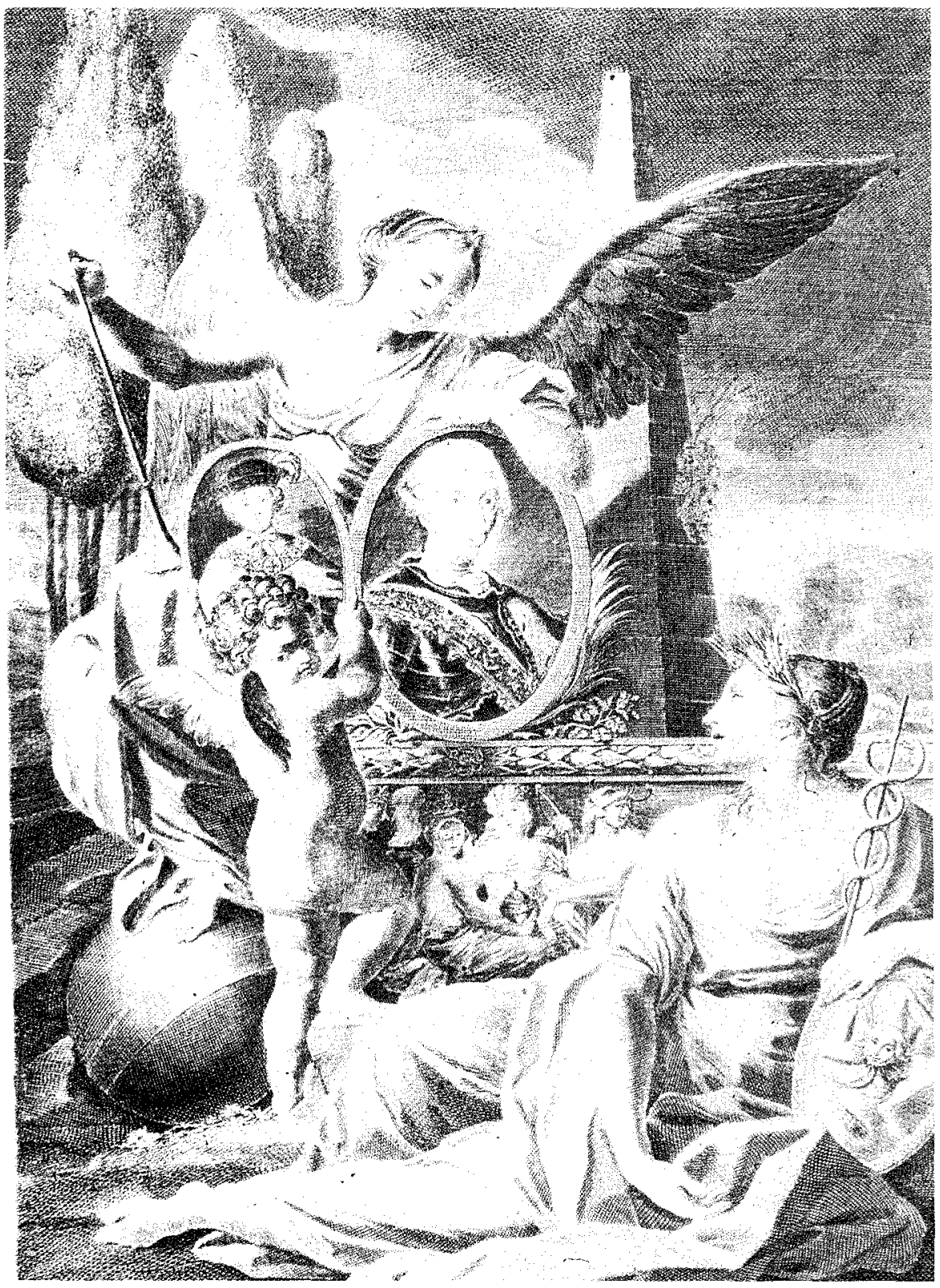

Fig. 2. Anteporta di Funerali per Carlo III Re delle Spagne e per I'Infante di Napoli D. Gennaro Borbone, Palermo 1789, firmata dal pittore Giuseppe Velasquez, incisione di G. Gramignani. 

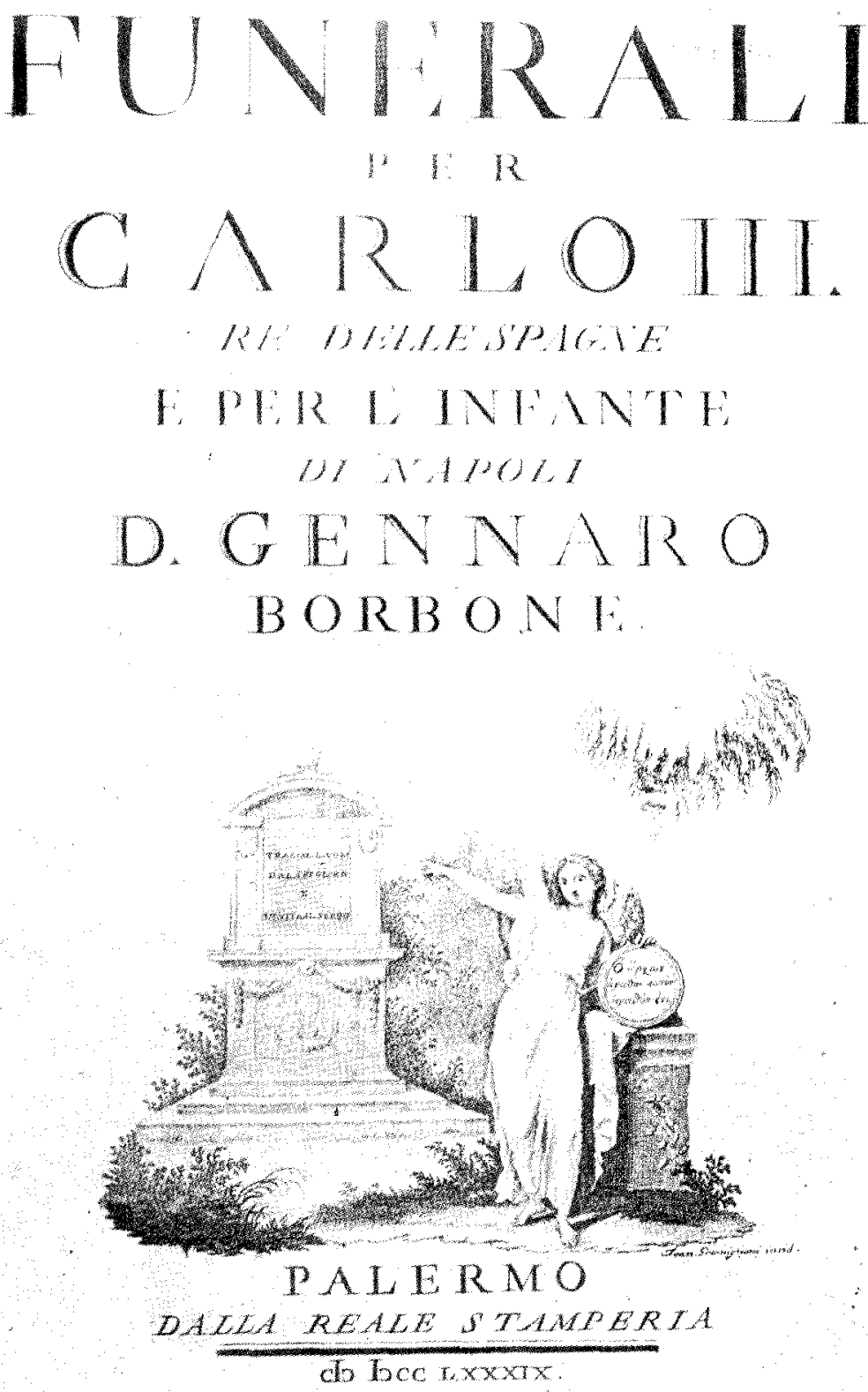

Fig. 3. Frontespizio di Funerali per Carlo III Re delle Spagne e per I'Infante di Napoli D. Gennaro Borbone, Palermo 1789. 
quale luogo ideale per la celebrazione delle esequie del re. A questa data infatti la chiesa si presenta ancora come un immenso cantiere che "stavasi fabbricando di nuovo, e comeché le interne muraglie fossero già compite, ma rozze, mancavano nondimeno la volta, il pavimento e la cupola, e le stesse pareti erano disadorne " ${ }^{3}$, a causa di quella «restaurazione», ancora non portata a termine, voluta ventidue anni prima dall'arcivescovo Serafino Filangeri ${ }^{4}$. I problemi che si frappongono alla realizzazione delle esequie di Carlo in Cattedrale sono notevoli; la spesa economica per sistemare in breve tempo il cantiere sembra non essere indifferente, ma la soluzione di optare per un'altra chiesa è altrettanto inaccettabile. Le alternative di Casa Professa, non abbastanza grande «quanto abbisognava per farvisi il mortorio con una pari splendidezza all'usata in quello di Filippo $V{ }^{5}$, e di $S$. Domenico, giudicata "straniera chiesa» ${ }^{6}$, non sono evidentemente soddisfacenti per l'arcivescovo Francesco Ferdinando Sanseverino e per l'ambizioso Tribunale del Real Patrimonio incaricato dal viceré Francesco d'Aquino, principe di Caramanico, di preparare le sontuose cerimonie funebri per le esequie di Carlo III e dell'Infante D. Gennaro Carlo.

L'incarico per la progettazione dei catafalchi e degli apparati decorativi delle chiese prescelte per i funerali reali viene affidato dal Maestro Razionale del Real Patrimonio, Giovanni Gioeni e Valguarnera, duca d'Angiò, al sacerdote d. Salvatore Attinelli, Ingegnere Camerale, e al capomastro Giovanni Giglio. La scelta del Maestro Razionale non è certo statá casuale; l'Attinelli sembra infatti essere il professionista più adatto ${ }^{7} \mathrm{a}$ realizzare l'ardita impresa che si sta attuando: svolgere i funerali di Carlo III

tutta la città nell'udirsi ciò, che si era risoluto di fare, e sopra gli altri se ne rallegravano all'estremo i vecchi cittadini, che per la lentezza necessaria, con cui si rifabbricava la Cattedrale, disperavano di poterla nella cadente loro età veder compita, e in questo modo la vedevano già rinascere». G.E. D। Blasi e Gambacorta, Relazione delle Pompe Funebri..., cit., pag. 25.

3 IVi, pag. 23.

4 Sulla vicenda del restauro della cattedrale si rimanda ai contributi di S. BoscARINO, G. CANTONe e M. GiufFRE, in: La Cattedrale di Palermo. Studi per lottavo centenario dalla fondazione, a cura di L. Urbani, Palermo 1993.

5 "Che si pratichi quello stesso che si praticò nella morte di Filippo V Padre del Re Carlo in tempo, che governava questi Regni». G.E. DI BLASI E GambacorTA, Relazione delle Pompe Funebri..., cit., pag. 23. Anche Carlo IV in Spagna impone per la stessa occasione sontuose funzioni da realizzarsi "según y en la misma forma que se hizieron por el Sr. Don Phelipe $V$.». In:V. Soto Caba, Los catafalcos de Carlos III: entre la influencia neoclásica y la herencia del barroco, in "Fragmentos", nn.12-13-14, junio 1988, pag. 134. In questo testo l'autrice avvia delle interessanti considerazioni sulla continuità stilistica che sussiste in Spagna fra i catafalchi eretti in onore di Filippo $\mathrm{V}$ e quelli per Carlo III.

6 G.E. Di Blasi e Gambacorta, Relazione delle Pompe Funebri..., cit., pag. 25.

7 «...Date avea bastante riprove de' suoi talenti...». Ibidem. I'Attinelli aveva d'altra parte lavorato in collaborazione con il Marvuglia durante tutta la travagliata vicenda del restauro, conosceva bene quindi le problematiche del cantiere. 
nel cantiere della Cattedrale. Per questa cerimonia l'Attinelli deve pensare non soltanto ad un catafalco reale da erigere sotto la cupola semicostruita, ma ad un vero e proprio progetto effimero di risistemazione dell'intero impianto della navata della chiesa, dal pavimento al soffitto. Alla luce di una lettura del progetto poi realizzato (sembra quasi interamente) ${ }^{8}$ secondo le idee di Ferdinando Fuga, che, si ricorda per inciso, era stato chiamato a Palermo nel 1767 per affrontare il progetto di restauro, la soluzione proposta dall'Attinelli si presenta come alternativa decisamente interessante e, per certi versi, anche innovativa.

L'importanza del disegno di questo architetto si rileva infatti non soltanto per l'ostinata e coraggiosa decisione di eseguire la cerimonia luttuosa in una chiesa impegnata in un lento rifacimento, ma soprattutto nell'ipotesi, che in questa sede analizzeremo, del suo porsi come alternativa al progetto di Ferdinando Fuga, secondo il cui disegno il cantiere stava (pur con difficoltà, e malgrado le opposizioni) procedendo. L'analisi delle due proposte avvia una prima riflessione sul possibile significato di un intervento come quello del 1789 in relazione al progetto del 1767; se si prende in considerazione il fatto che la presentazione del tempio più importante della città secondo il disegno dell'ingegnere Attinelli (al di là dell'occasione del funerale del monarca) possa nascondere in realtà la sottile e velata decisione di promuovere un progetto diverso da quello del modello lasciato dal Fuga durante il soggiorno del 1767, non si può non valutare questo intervento come un nuovo spunto di lettura per il lungo e travagliato cantiere della cattedrale, sottolineando che si potrebbe trattare dell'inquietante ipotesi che il progetto effimero per il funerale di Carlo III altro non sia che l'ultimo tentativo del Tribunale del Real Patrimonio di veder realizzato (e quindi implicitamente «pubblicizzato») il controprogetto alternativo al disegno dell'architetto toscano.

Sull'esistenza di un progetto alternativo, di cui però non è stato ancora trovato alcun documento che ne provi la veridicità, ci fornisce interessanti notizie il canonico Stefano Di Chiara vissuto contemporaneamente agli avvenimenti relativi al cantiere della Cattedrale alla fine del XVIII secolo. Questo personaggio, considerato dai suoi contemporanei un erudito, scri-

\footnotetext{
8 Come ha sottolineato anche M. GIUfFrÉ, lo storiografo Di Blasi afferma che «...si dié mano all'opera...fatti solo certi piccoli cambiamenti...». Ivi, pag. 42. Ed anche: M. GIUFFrÉ, // cantiere della Cattedrale di Palermo da Ferdinando Fuga a Emmanuele Palazzotto...,cit., pag. 260. II canonico S. Di Chiara sottolinea che nel corso del restauro gli architetti avevano " riformato alcuni difetti, com'era quello del cornicione centinato, e delle nicchie disposte nel cilindro della cupola, che la rendevano mal sicura, e pericolosa, e altre cose siffatte". S. DI CHIARA, Discorso istorico critico sopra le chiese maggiori e cattedrali in questa città di Palermo, Palermo 1825, pag. 32.
} 


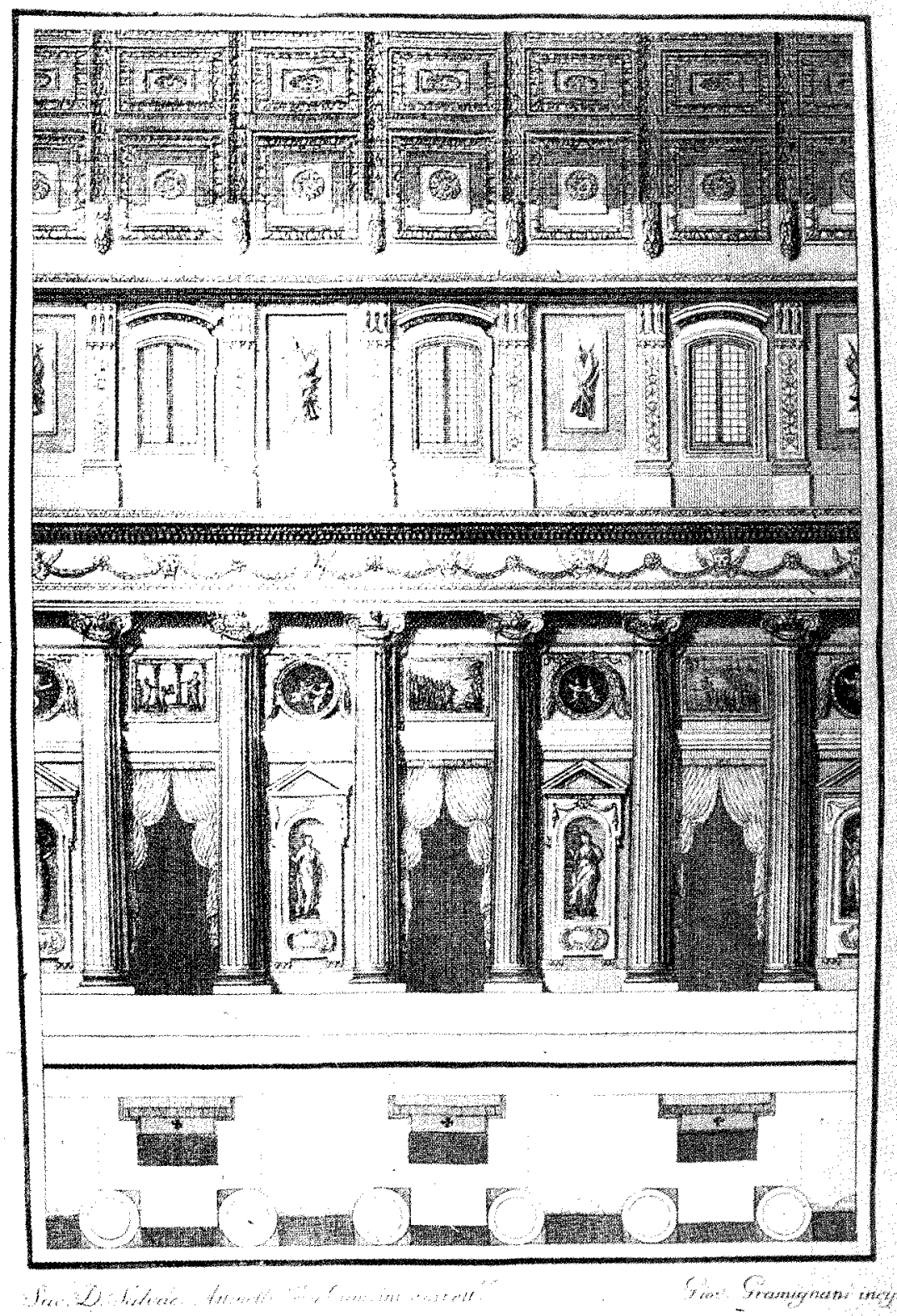

Fig. 4. Apparato della navata centrale della cattedrale di Palermo, realizzato in occasione delle esequie di Carlo III, incisione di G. Gramignani, disegno di S. Attinelli (in Funerali per Carlo III Re delle Spagne e per l'Infante di Napoli D. Gennaro Borbone, Palermo 1789, tav. IV.). 
ve nel 1825 un opuscolo ${ }^{9}$ in cui traccia un'analisi storica degli avvenimenti che caratterizzano la lunga vicenda della Cattedrale di Palermo. II canonico riferisce che nel 1777, dieci anni dopo il breve soggiorno di Fuga a Palermo, i problemi che si frappongono alla realizzazione del «restauro» secondo i suoi disegni sembrano ancora essere insormontabili: oltre alla mancanza dei fondi economici necessari, la deputazione dei nove soggetti, eletti dal governo per occuparsi della vicenda dell'«ammodernamento" della Cattedrale, sembra invece in tutti i modi voler intralciare il progetto «al disegno del Fuga aperta guerra dichiarando» 10. Tra questi deputati, Alessandro Vanni, Principe di S. Vincenzo, che «nelle cose di architettura sommamente valea» ${ }^{11}$, insieme all'architetto Giuseppe Venanzio Marvuglia, ancora agli esordi della sua lunga e valorosa carriera, in accordo con il resto della deputazione, "scorrettissimo il disegno di Fuga riconobbero, e principalmente due gravi abbagli vi riprovavano ${ }^{12}$. In primo luogo l'uso di pilastri di ordine corinzio e colonne binate sempre dello stesso ordine che sorreggono gli uni l'architrave e le altre gli archi «ingarbugliando» e «travolgendo" la composizione. In secondo luogo la critica è rivolta alla cupola corinzia disegnata dal Fuga che «colla facciata gotica della chiesa non combinava» ${ }^{13}$ esibendo «una sconcordia, che alla vista de' più rozzi del popolo non sarebbe sfuggita" ${ }^{14}$. Spedite a Napoli replicate lamentele, la deputazione chiede al re di ordinare un altro disegno «più corretto, e più confacente alla primaria chiesa della Sicilia» ${ }^{15}$. La scontentezza e l'indecisione non sono certo una novità; come è noto, nel 1752, l'architetto Giovan Battista Vaccarini viene chiamato per una consulenza sul restauro della cattedrale; anche in questo caso, pur non conoscendo le intenzioni progettuali dell'architetto, (né abbiamo la certezza sulla sua redazione di un disegno), sappiamo che ben presto viene allontanato dal cantiere per essere sostituito quindici anni dopo dal rivale Ferdinando Fuga ${ }^{16}$. Neanche per l'architetto toscano i rapporti con la deputazione locale sono facili, sembra anzi che nello stesso periodo si invitasse Luigi Vanvitelli a fornire una

\section{lbidem.}

10 IVi, pag. 28.

11 Ibidem. Sulla personalità di questo nobile che le recenti ricerche vanno sempre più delineando si veda anche: G. LEONE, / Collegio di Maria a Monreale e Alessandro Vanni "architetto": un'ipotesi per un progetto, in «Lexicon» n. 0 , in c. d. s.

12 S. DI CHIARA, op. cit., pag. 28.

13 Ivi, pag. 29

14 lbidem.

15 lbidem.

16 Per un ulteriore approfondimento dei rapporti fra G.B. Vaccarini e Ferdinando Fuga si confronti: M. R. NoBlLe, I volti della «Sposa», Le facciate delle Chiese Madri nella Sicilia del Settecento, Palermo 2000, pagg. 41-42. 




Fig. 5. Catafalco eretto allinterno della cattedrale di Palermo in occasione delle esequie di Carlo III, incisione di G. Gramignani, disegno di S. Attinelli (in Funerali per Carlo III Re delle Spagne e per l'Infante di Napoli D. Gennaro Borbone, Palermo 1789, tav. V). 
sua proposta alternativa a quella del rivale Fuga. ${ }^{17}$ Secondo le notizie riportate dal Di Chiara, sono gli stessi deputati a proporre in questa occasione un nuovo progetto; in particolare vengono coinvolti it Principe di $\mathrm{S}$. Vincenzo e il Marvuglia che sottolineano come il loro disegno sia non soltanto più confacente alle buone regole d'architettura, ma molto meno dispendioso di quello dell'architetto toscano. Si decide dunque di far realizzare un modello, così come era stato fatto per il progetto Fuga, e di spedirlo a Napoli con una rappresentanza affinché il re possa decidere quale dei due meriti la vittoria. Ferdinando di Borbone stabilisce che una giuria composta dai più rinomati architetti napoletani ${ }^{18}$ giudichi quale sia il disegno più corretto da eseguire, e questi (contrariamente alle aspettative dei palermitani) decidono all'unanimità in favore del disegno del Fuga, sottolineando tra l'altro che, diversamente da quanto richiesto dalla deputazione, non vi è bisogno di apportare nessuna correzione. A questo punto si comanda che il progetto vincitore sia eseguito immediatamente. Malgrado «l'oracolo» del re, i deputati continuano a lamentarsi, e con ogni scusa a perdere tempo. L'arcivescovo Francesco Ferdinando Sanseverino spinge invece per iniziare i lavori e offre persino di caricarsi di un ulteriore onere finanziario affinché il cantiere cominci subito. Nel 1780 gli ordini del re non sono ancora stati eseguiti, tanto che questi è indignato per non essere stato immediatamente ubbidito; per punire l'atteggiamento ostile dei palermitani, e soprattutto per placare il coro di critiche che rallentano la fabbrica, decide di ridurre il numero dei deputati a tre: il Principe di S. Vincenzo, l'abate Luigi Gravina e l'avvocato fiscale del patrimonio, Michele Maria Perramuto; lasciando però la facoltà ai suddetti restanti deputati di decidere quale architetto assumere per l'esecuzione dei disegni del Fuga. G. V. Marvuglia è l'architetto prescelto. Se con questa azione si è inteso eliminare i problemi di contestazione, sicuramente un errore di valutazione è

\footnotetext{
17 IVi, pag. 41 e pag. 50 (nota). L'autore si riferisce ad una lettera di Luigi Vanvitelli in cui l'architetto afferma: «ll marchese Soragna, nepote del Fogliani, molte cose dissemi sopra la Chiesa di Palermo per farmi fare un disegno, ma non veggo risultato, benché l'affare non sarebbe maturo adesso". Cfr. Le lettere di Luigi Vanvitelli della Biblioteca Palatina di Caserta, a cura di F. Strazzullo, Galatina 1976, III, n. 1328 (Napoli, 21 aprile 1767).

18 Una giuria di architetti fu sicuramente composta nel 1780 su ordine del re, almeno per esaminare il progetto di Fuga. Fra gli esperti vi è pure Carlo Vanvitelli, figlio di Luigi, per questo Fuga si preoccupa dell'esito di questo giudizio: «...So che...è stata ordinata questa revisione, forse per sbaglio, all'arch. Cav. Carlo Vanvitelli, acciò veda, riconosca e riferisca quanto passa su quest'assunto, onde supplico la bontà di V. E. a rimediare ad un tale disordine, il quale molto pregiudizierebbe alla mia stima ed onore, il quale apprezzo più della vita stessa...». N. BASILE, La Cattedrale di Palermo: l'opera di Ferdinando Fuga e la verità sulla distruzione della tribuna di Antonello Gagini, Firenze 1926, pagg. 42-43; per un ulteriore approfondimento della vicenda si veda il contributo di G. CANTONE, II progetto di Ferdinando Fuga...,cit, pag. 143.
} 
stato fatto sulle personalità dei soggetti della deputazione rimasti; sembra infatti che proprio questi saranno i protagonisti della misteriosa vicenda di cui diremo in seguito

Fin qui le notizie fornite dal Di Chiara sull'esistenza di un controprogetto. II canonico (sottoliniamo ancora una volta che si tratta di un personaggio tenuto in grande considerazione dai suoi contemporanei) è però smentito nel 1926 da uno storico, Nino Basile, che nella sua opera sulla cattedrale ${ }^{19}$, incentrata fondamentalmente su una appassionata difesa dell'operato di Fuga, basandosi su alcuni errori di date e su alcune incertezze dell'autore, dichiara erronee le tesi sostenute dal canonico nel 1825, e quindi di conseguenza anche infondata l'esistenza di un disegno alternativo a quello di Fuga, sottolineando tra l'altro che non esistono documenti che anche soltanto accennino al controprogetto Vanni-Marvuglia. Non si può però non riflettere, a difesa del canonico Di Chiara che, se i documenti non trattano esplicitamente l'argomento di un nuovo progetto, ad un'attenta lettura, neanche lo smentiscono. Si rifletta ad esempio su questo brano:«...quanto al disegno di tal nuova fabbrica la M. S. ha scelto già ed approvato quello del cav. Fuga..." ${ }^{20}$; in cui sembra che "quello del» presupponga che il disegno di Fuga sia stato scelto fra altri. Oppure ancora: "l disegni ed il modello di questa restaurazione rimasti in Palermo furono tempo fa chiamati dal re in occasione del progetto avanzato da Capitolo, il quale diceva di essere molta eccessiva la spesa secondo i disegni del Cav. Fuga e che potevasi di molto risparmiare quando si fosse fatta la restaurazione di quel tempio in altra maniera differente e che era stata proposta dallo stesso Capitolo. Ma non avendo passata per il canale della Giunta questa controversia, sappiamo che il re l'avesse risoluta con prescriversi di eseguirsi a puntino i disegni e le relazioni del Fuga" ${ }^{21}$. Si sottolinea in questo caso che esisteva un modello di restaurazione proposto dallo stesso Capitolo. Ed infine: «... i disegni ed il modello (del Fuga)...furono... di là chiamati in occasione di avere il Capitolo di quella Chiesa Cattedrale progettato che potevasi di molto risparmiare l'ingentissima spesa di quella restaurazione, quando si fosse fatta non già secondo i disegni del cav. Fuga, ma secondo le variazioni proposte e pensate dallo stesso Capitolo... "22. Al di là dell'interpretazione di questi documenti, quel che ci sembra doveroso sottolineare è la mancanza delle motivazioni che avrebbero portato il canonico Di Chiara a

\footnotetext{
19 Per l'opera di N. BASILE si rimanda alla nota 18.

20 Ivi, doc. XIV, pag. 137.

21 Ivi, doc.XV, pag. 139

22 Ivi, doc. $X v$, pag. 140
} 
inventare l'esistenza di un controprogetto; è pur vero che nessun altra fonte del periodo offre informazioni decisive che confermino l'esistenza di un disegno alternativo. Appare del resto indicativo che un cronista come il Di Marzo Ferro definisca il canonico come «onore di Palermo non solo, ma di tutta la Sicilia», ${ }^{23}$ sottolineandone così la serietà. Se si ipotizza che l'esistenza di un progetto in cui sono coinvolti Vanni e Marvuglia sia reale, un ulteriore quesito da sciogliere si presenta: sarebbe interessante capire cioè che tipo di proposta avrebbe potuto formulare la deputazione. Pur non esistendo materialmente i disegni (come d'altra parte non sono pervenuti neanche quelli di Ferdinando Fuga), ${ }^{24}$ in alcuni documenti ritrovati sono presenti i suggerimenti del Principe di S. Vincenzo e del Marvuglia che propongono, in alternativa ai pilastri di ordine corinzio del progetto Fuga, un ordine gigante di colonne "...com'era ben facile in un paese di marmi abbondevole, grandiose colonne a sostentar l'architrave" ${ }^{25}$. Questa soluzione non avrebbe permesso però il reimpiego delle antiche colonne, ma sembra che già da tempo non si sentisse più questa necessità ${ }^{26}$. Le altre critiche riguardano, come è stato già accennato, la cupola che presumibilmente volevano «meno alta e non di ordine corin$z i 0 »{ }^{27}$. Inoltre «i deputati della fabbrica vogliono evitare il rivestimento di marmi policromi, ufficialmente per ridurre i costi dell'esecuzione...in realtà per aderire alle richieste di Marvuglia e di Attinelli» ${ }^{28}$, i quali premono affinché i marmi di gusto "gotico», previsti dal Fuga siano sostitutiti con un rivestimento a stucco. Secondo i deputati, infatti, l'apparato di marmi non avrebbe reso possibile la creazione di quel "disegno unitario» per cui si era deciso un «ammodernamento» della fabbrica, altrimenti si sarebbe corso il rischio che, seguendo le indicazioni del Fuga, la chiesa sarebbe restata "parte coll'antica Gotica architettura e parte colla nuova Architettura sul gusto romano" ${ }^{29}$ e perciò a lavori ultimati sarebbe risultata «irregolare la ristaurazione di quel tempio» ${ }^{30}$.

23 G. Di Marzo Ferro, Guida istrtuttiva..., Palermo 1858, pag. 627 (nota).

24 E' attraverso il contributo del Villabianca, che fornisce le uniche copie del progetto di Fuga, che siamo a conoscenza delle preziose informazioni riguardanti il disegno la cui copia autografa è andata perduta.

25 S. DI CHIARA, Discorso istorico...cit, pag. 29.

26 Da un confronto con gli altri apparati effimeri eretti in cattedrale nel xvili secolo è possibile constatare che le colonnine originali della navata centrale che sostenevano gli arconi in cattedrale venivano regolarmente mascherate dietro pilastri. Si dovrebbe dunque riflettere che forse ques ta soluzione compositiva non era molto apprezzata.
27 G. CANTONE, op.cit, pag. 147.
28 IVi, pag. 143.
29 N. BASILE, op. cit., doc.V, pag. 128.
30 Ibidem. 
L'ipotesi che si vuole avviare in questa sede è che i funerali del 1789 sono forse l'occasione ideale per proporre un'ultima volta al re un progetto che probabilmente non è stato mai veramente posto alla sua attenzione. In questa occasione, non saranno i disegni o il modello ad essere mostrati ma un apparato effimero che riproduca le idee progettuali della Deputazione ${ }^{31}$. Il Tribunale è incaricato dal viceré di occuparsi dei funerali ed il presidente è ancora una volta D. Michele Perramuto ${ }^{32}$. L'architetto scelto è $\mathrm{d}$. Salvatore Attinelli, un personaggio che è stato presente negli anni cruciali della vicenda ma che non si è esposto come il Marvuglia, non essendo tra l'altro il suo nome legato al progetto bocciato. L'occasione unica che si presenta ai deputati sembra irrinunciabile, anche per questo forse non si bada a spese, e si preferisce affrontare un' impresa "ardita» piuttosto che scegliere un'altra chiesa dove sicuramente l'impegno economico sarebbe stato di molto inferiore. A sostegno di queste considerazioni, si rifletta sulla motivazione che spinge l'Attinelli a scartare immmediatamente l'ipotesi di una proposta aderente al progetto dell'architetto toscano (avrebbe potuto infatti utilizzare le parti già esistenti) ${ }^{33}$, proponendo invece un progetto in cui l'apparato decorativo effimero stravolge, ricopre e nasconde le parti della struttura già realizzate. In considerazione del fatto che il tempo a disposizione è molto poco, appena tre mesi, inframezzati fra l'altro dalla progettazione dei catafalchi per i funerali reali del piccolo Gennaro nella Casa Professa e nella Cappella di San Pietro a Palazzo Reale (qui eseguiti in onore sia dell'Infante di Napoli che dello stesso Carlo III) ${ }^{34}$, la proposta dell'apparato effimero dell'Attinelli appare più che coraggiosa, politicamente ben motivata, rappresentando la soluzione ideata contro il modello da con-

31 «Si alzò una novella chiesa di diversa architettura e di altra idea...per la moda oggi co. rrente...che vuole tutto eseguirsi a disegno di greca architettura, cangiata mirossi la Cattedrale in una vasta Galleria...non credea frattanto ogni uno di vedere al solito un luttuoso apparato, ma vi ammirava un reale e naturale edificio...". F. M. EMANUELE E GAETANI, Le feste reali...cit., pag. 192.

32 Funerali per Carlo III...,cit., p. III. II nome del principe di S. Vincenzo non appare nel libro dei funerali di Carlo, ma è sicuramente ancora dietro questa vicenda; in questa occasione suo fratello minore, Francesco, vescovo di Cefalù, é chiamato a tenere «le cinque solenni assoluzioni» per l'Infante di Napoli insieme ad altri tre alti prelati e all'arcivescovo Sanseverino. Ivi, pag. 32.

33 Come riferisce il Di Blasi erano state già «innalzate le pareti, i pilastri, e gli archi, e una porzione di cupola...». Ivi, pag. 42.

${ }^{34}$ II Tribunale del Real Patrimonio camerale sostenne le spese per tre funerali: il primo da tenersi con un Triduo a Casa Professa per le sole esequie del principe D. Gennaro; il secondo nella Cappella di S. Pietro a Palazzo Reale dove fu stabilito un Triduo nei giorni 4-5-6 marzo per l'Infante e subito dopo un secondo Triduo in onore di Carlo, utilizzando lo stesso catafalco (le iscrizioni e l'effige di Gennaro furono sostituite con quelle relative al re, come pure furono aggiunti i simboli del monarca), il terzo, il più grandioso si organizzò per Carlo in cattedrale dove furono tenuti i Novendiali a partire dal 28 aprile 1789. 


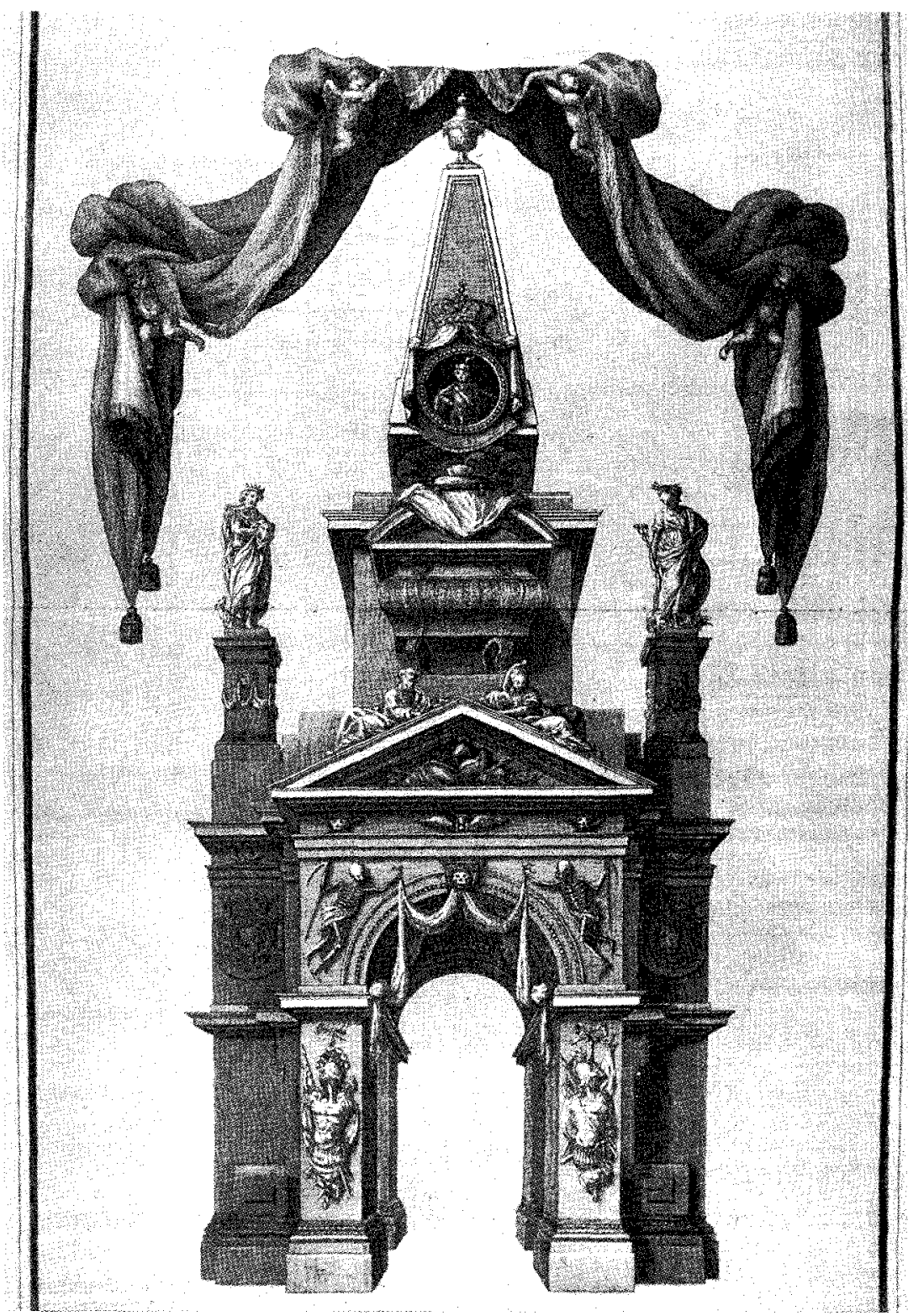

Fig. 6. Catafalco eretto all'interno della Cappella di S. Pietro a Palazzo Reale in occasione delle esequie di Carlo III e di Gennaro di Borbone, incisione di G. Gramignani, disegno di S. Attinelli (in Funerali per Carlo III Re delle Spagne e per l'Infante di Napoli D. Gennaro Borbone, Palermo 1789, tav. III). 
testare. Nessuno può ignorare in questo caso la volontà e l'ambizione di proporre ancora una volta il disegno che era stato bocciato, promuovendolo tra l'altro attraverso la pubblicazione delle incisioni in un libro dedicato al re Ferdinando. Se cosi non fosse, bisognerebbe soffermarsi a riflettere sui particolari compositivi del progetto effimero dell'Attinelli. L'architetto prevede per la navata un'interessante proposta che la trasforma in un luogo gentilizio di sepoltura dei principi, un vero e proprio santuario della famiglia reale, un pantheon che sottolinei i legami e la fedeltà di Palermo alla Corona. Nei sedici intercolumni, otto per fianco, l'apparato decorativo è studiato in modo da far immaginare allo spettatore la presenza degli avelli reali e degli altari per celebrare le messe private sull'esempio del S. Lorenzo a Firenze o del S. Lorenzo dei P.P. Gerolamini nell'Escurial (cosi come suggerisce il «regio istoriografo»D. Giovanni Evangelista Di Blasi.) ${ }^{35}$. Per l'ordine principale della navata Attinelli sceglie l'ordine gigante di colonne ioniche (e subito il Di Blasi sente di dover giustificare il progettista sottolineando che è stato utilizzato l'intercolumnio di quest'ordine quale è raccomandato dai maestri di «Scienza Architettonica» come Vitruvio e Palladio per essere uno dei più belli ed eleganti) ${ }^{36}$. Si ricorda a questo punto che contro il progetto di Fuga la deputazione aveva proposto l'impiego di colonne giganti di marmo, e il Di Chiara, a favore della proposta dei deputati, così si era espresso: «... la chiesa diverrebbe tanto più maestosa quanto più svelta $\mathrm{e}$ semplice, e mostrerebbe al primo ingresso dalla porta maggiore tutta se stessa; e tutto il suo ordine architettonico a gli occhi de' riguardanti» ${ }^{37}$. Inoltre, come è stato già sottolineato, i deputati trovavano scorretto l'uso dell'ordine corinzio (e qui viene proposto lo jonico).

Quanto meno si tratta di una concidenza interessante; se non vogliamo credere che il Tribunale abbia sfruttato questa luttuosa occasione per riproporre il suo progetto, dobbiamo almeno sottolineare che è verosimile che l'Attinelli progetti un impianto che utilizza le idee di un disegno che forse già conosceva. Se così fosse, sembra plausibile pensare che dietro la figura di Attinelli ci sia una personalità molto più forte in grado di escogitare questo stratagemma per proporre al pubbblico un'alternativa al progetto che si sta realizzando ${ }^{38}$.

\footnotetext{
G. e. DI BLASI, Relazione delle Pompe Funebri...,cit., pag. 47. Ibidem.

S. DI CHIARA, op. cit, pag. 29.

II DI BLASI sottolinea che il " genio produttore" dell'idea è il duca d'Angiò e che l'Attinelli trova "eseguibili" le sue idee; per questa ragione merita un elogio alla sua perizia. G. E. Dı BLASI e GambacuRTA, op.cit., pag. 40.
} 


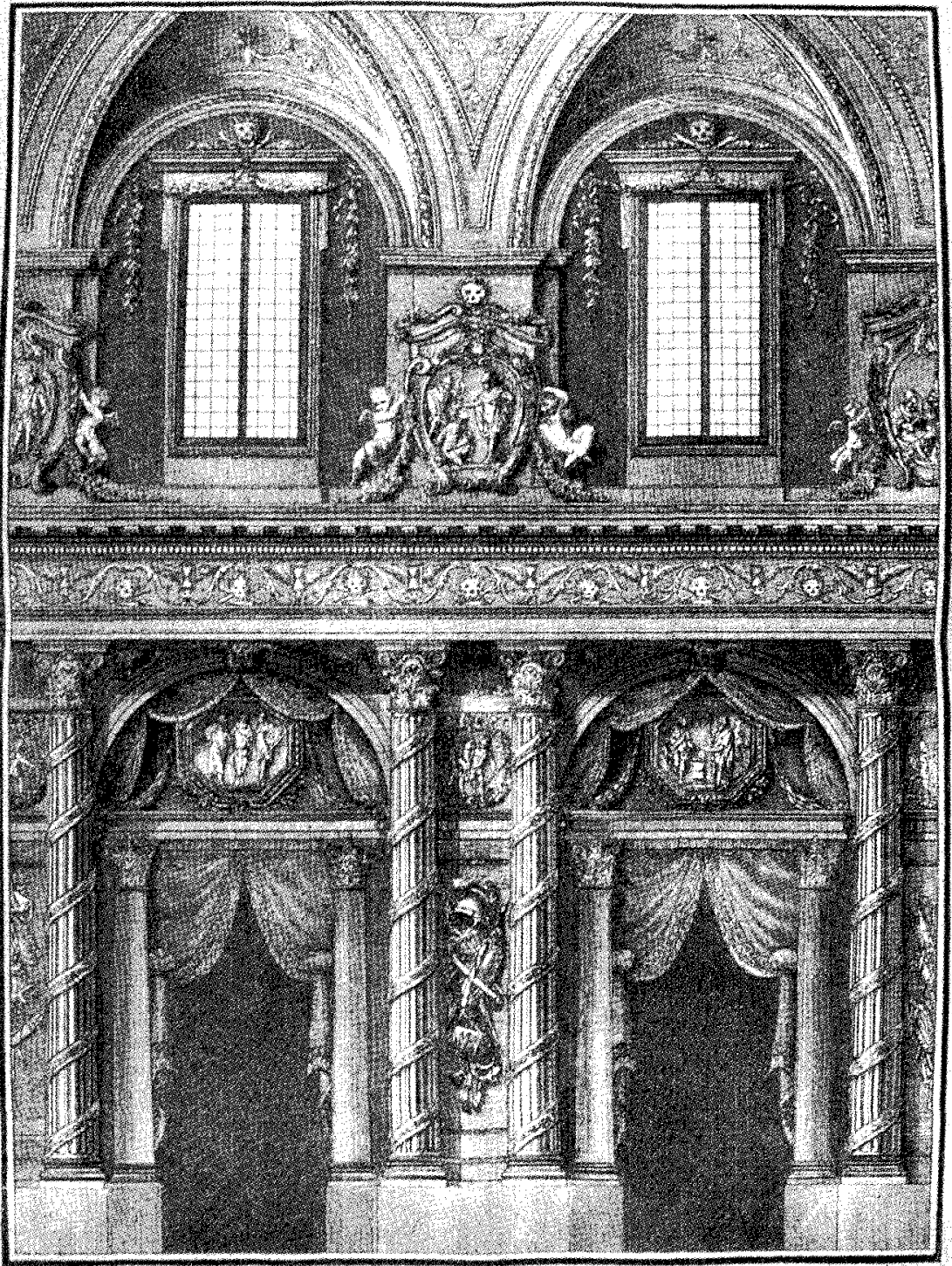

W. 2 , w f

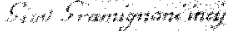

Fig. 7. Apparato eretto nella navata centrale di Casa Professa in occasione delle esequie dell'Infante $D$. Gennaro di Borbone, incisione di G. Gramignani, disegno di S. Attinelli (in Funerali per Carlo III Re delle Spagne e per IInfante di Napoli D. Gennaro Borbone, Palermo 1789, tav. I). 
Nell'apparato effimero del 1789 viene realizzata una composizione in cui si alternano nel ritmo delle colonne delle finte celle e delle nicchie. In queste sono inserite delle statue, in mistura di gesso e polvere di marmo, che rappresentano le virtù del monarca. Sopra ogni nicchia un circolo con due puttini con simboli e geroglifici allusivi alla statua. Sopra ogni cella invece quadroni a bassorilievo esprimono le gesta del sovrano.

In corrispondenza delle grandi colonne dell'ordine principale, Attinelli propone un secondo ordine di pilastri attici, fra cui si alternano negli spazi maggiori le finestre (sopra le celle) e in quelli minori (sopra le nicchie) bassorilievi, trofei militari e insegne reali. Collabora nelle esecuzioni delle parti pittoriche e decorative Giuseppe Velasquez.

Per la volta il progetto 1789 prevede un disegno suddiviso a riquadri di diverse grandezze mentre un "solarone» di legno avrebbe mascherato la mancanza del pavimenio. Tutto nell'insieme era studiato affinché l'apparato effimero sembrasse realizzato in nobili e preziosi materiali.

Si potrebbe a questo punto obiettare che questa ultima scelta è in contrapposizione alle critiche fatte al Fuga che proponeva nel suo progetto I'utilizzo di una mistura di marmi. I deputati si erano espressi con un parere nettamente sfavorevole a soluzioni come questa, ritenute estrose oltre che estremamente costose. Come ha sottolineato S. Boscarino, ${ }^{39}$ la personalità dei direttori dei lavori, l'architetto Marvuglia e l'ingegnere Salvatore Attinelli, erano assolutamente diverse da quella del Fuga; l'ostinato rifiuto delle finiture delle superfici in marmi policromi e la preferenza verso quelle a stucco (sulle quali venivano distesi i colori, tipici delia decorazione neoclassica, azzurro e bianco), oltre alle ragioni economiche mette in luce il diverso spirito dei due progettisti. Queste ragioni confermerebbero il controsenso della scelta dell'Attinelli. La critica dei deputati al Fuga si era basata, però, sulla considerazione che egli avesse redatto un progetto senza prevedere una stima dei costi, per cui la polemica faceva leva soprattutto sui punti in cui il progetto risultava più costoso (quindi nell'utilizzo dei marmi policromi). Questa potrebbe essere la spiegazione che motiverebbe le ragioni che spinsero il progettista a riformulare una proposta così pesantemente criticata ventidue anni prima.

Nella stessa tavola del libro dedicato al funerale di Carlo, in cui è inciso l'apparato per la navata centrale, è presente un particolare che, alla luce di queste osservazioni, si riveste di nuovi inquietanti significati: sotto l'alzato è riprodotta una porzione di pianta dell'apparato (nelle altre tavole non vi è

39 S. Boscafino, op. cit., pag. 99. 




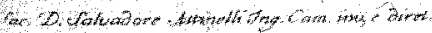

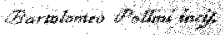

Fig. 8. Catafalco eretto in occasione delle esequie dell'Infante D. Gennaro di Borbone, incisione di G. Gramignani, disegno di S. Attinelli (in Funerali per Carlo III Re delle Spagne e per l'Infante di Napoli D. Gennaro Borbone, Palermo 1789, tav. (I). 
nulla di simile). Perchè mai si era sentita la necessità di fornire ulteriori informazioni sulla composizione, sottolineando anche con una sezione orizzontale la presenza di colonne giganti?

L'ultima tavola del libro (la seconda che riguarda gli apparati eseguiti in cattedrale) propone invece il particolare del catafalco reale sotto la crociera. Si tratta di un'incisione di grande formato in cui è possibile leggere un impianto formato da quattro arconi a pieno centro; questi sembrano sostenuti dallo stesso tipo di composizione che caratterizza la navata, cioè l'ordine gigante di colonne che sostiene un secondo basso ordine di pilastri attici. Le colonne del primo ordine sono però binate e sembrano venire avanti rispetto a un ordine anch'esso binato di paraste joniche, formando insieme una struttura in grado di sostenere i pesanti arconi. A differenza delle colonne della navata centrale queste sono sostenute da un alto basamento che fa da zoccolo anche per le paraste retrostanti. Sopra gli arconi si alza un alto tamburo caratterizzato da coppie di paraste binate (probabilmente anche queste joniche) inframezzate da grandi finestre.

Nel centro della crociera, sotto la cupola, una superba struttura neoclassica rappresenta il catafalco del re. Sopra un grande piedistallo composto da un alto basamento (forato da un apertura affinché i celebranti possano raggiungere l'altare maggiore) si alzano otto colonne isolate con basi e capitelli corinzi (si noti che la scelta del tanto criticato ordine corinzio avvalorerebbe l'ipotesi che il catafalco non sia stato progettato contemporaneamente all'apparato della navata) che racchiudono l'urna reale sostenuta da quattro leoni.

Sopra il cornicione un'edicola coronata da frontespizio triangolare accoglie la statua del sovrano; ai fianchi di questa piccola struttura templare si innalzano due alte e slanciate piramidi, mentre nella parte retrostante, su cinque gradini circolari, quattro atlanti sostengono il globo terrestre adornato con i gigli della famiglia Borbone. Numerose statue alludenti sia alle qualità del re che ai legami tra il regno di Sicilia e la famiglia reale arricchiscono ulteriormente il mausoleo.

L'immagine architettonica è immediatamente percepibile come struttura aderente alle nuove istanze del linguaggio dell'epoca, sebbene, per certi versi, il gusto per il particolare ricercato ed in generale per una ricca decorazione sia ancora molto evidente. Se messo in relazione con un catafalco decisamente neoclassico come quello che, per la stessa occasione, è innalzato a Roma dall'architetto Pannini in San Giacomo degli Spagnoli ${ }^{40}$,

40 G. PANNINI è l'architetto a servizio dell'ambasciata spagnola a Roma. Come ha sottolineato $\checkmark$. Soto $\mathrm{CABA}_{\mathrm{A}}$ il mausoleo romano è "un ejemplo único, un modelo insólito que representa, de 
l'apparato dell'Attinelli risulta più complesso nei suoi effetti pittorici e chiaroscurali legati ancora ai retaggi iconografici della tradizione locale. II mausoleo romano è infatti un vero e proprio tempio dorico, molto più austero nel suo rigoroso classicismo rispetto al catafalco palermitano. Questo risulta iconograficamente lontano anche dalla versione fornita per la stessa occasione da Carlo Vanvitelli nella Real Cappella di Napoli, dove viene eretto un catafalco monolitico di pianta ottagonale con pilastri dorici ${ }^{41}$. Altrettanto interessante risulta il confronto con il catafalco catanese innalzato nella chiesa della confraternita dei Bianchi nel maggio del $1789 \mathrm{su}$ progetto di Léon Dufourny. Questo mausoleo è composto da un basamento (anch'esso forato da un arco per permettere il passaggio ai celebranti) impreziosito da ornamenti dorati e argentati. Due bassorilievi alludenti alla Giustizia e alla Storia che scrive le gesta del sovrano serrano l'arco. Sopra questo un'alta piramide a gradoni, composta di cristalli e illuminata a giorno, simboleggia l'immortalità del sovrano, rafforzata dalla presenza di una Fenice sull'estremità dell'apparato, mentre ai piedi di questa un prezioso sarcofago rappresenta l'urna del defunto. Ai lati due alte colonne (simboleggiano quelle d'Ercole) fiancheggiano il mausoleo. Anche in questo caso, come è immediatamente percebibile, siamo di fronte ad una struttura effimera molto diversa clal catafalco palermitano.

La lettura iconografica delle varie e diverse versioni dei mausolei innalzati per i funerali di Carlo III avvia una considerazione sul gusto e sulle tendenze del linguaggio architettonico nelle diverse città del regno, dove sicuramente le istanze del Neoclassicismo vengono recepite in maniera diversa e vengono fortemente influenzate sia dalle tradizioni locali che dalla personalità dei progettisti che vi lavorano. Anche per queste ragioni non si può non riflettere sull'importanza che rivestono le incisioni del libro pubblicato per la morte di Carlo III nel loro offrire uno spaccato del pensiero architettonico dell'epoca; gli anni in cui viene celebrato questo funerale, così come tutto il periodo in cui si inserisce l'intera problematica vicenda della «restaurazione» della cattedrale, sono infatti anni cruciali per il dibattito architettonico che, come è leggibile dalle stesse strutture effimere, sembra subire ancora forti oscillazioni di gusto.

Certamente gli interrogativi che avvolgono la vicenda del «controprogetto» appaiono numerosi, così come sono ancora molti i nodi da sciogliere (in parte

forma ortodoxa, la estética neoclásica, acorde con aquellos autores partidarios de un clasicismo que responda a la obras antiguas y al legado de la civilización griega...». V. SоTo CABA, Catafalcos reales del Barroco Español. Un estudio de arquitectura efimera, U.N.E.D., Madrid 1991, pag. 355.

41 Per alcune considerazioni sul catafalco napoletano si veda: $/ V i$, pag. 356. 
irrisolvibili per la drammatica perdita dei materiali archivistici della cattedrale, distrutti nell'incendio del 1860). II testo pubblicato e le incisioni qui contenute sembrano pertanto offrire uno spiraglio su un argomento decisivo per la Sicilia del tardo XVIII secolo. Le interazioni tra politica, cultura, rappresentazione e architettura sembrano trovare nei funerali di Carlo III un nodo di scontro, e di conflitto, sinora solo vagamente intuibile, ma che ulteriori studi dovranno necessariamente dirimere. 